\title{
Caffeine and Reactive Hyperemia
}

\section{Gary F. Merrill*, Denisa M. Costea, Victoria A. Sharp}

Department of Cell Biology and Neuroscience, Division of Life Sciences, School of Arts and Sciences, Rutgers University, Nelson Biological Laboratories, Piscataway, NJ, USA

Email: *merrill@dls.rutgers.edu

How to cite this paper: Merrill, G.F., Costea, D.M. and Sharp, V.A. (2019) Caffeine and Reactive Hyperemia. World Journal of Cardiovascular Diseases, 9, 437-448. https://doi.org/10.4236/wjcd.2019.97039

Received: May 9, 2019

Accepted: July 15, 2019

Published: July 18, 2019

Copyright $\odot 2019$ by author(s) and Scientific Research Publishing Inc. This work is licensed under the Creative Commons Attribution International License (CC BY 4.0).

http://creativecommons.org/licenses/by/4.0/

\begin{abstract}
The physiological/pathophysiological effects of caffeine on the human cardiovascular system have not been investigated by physiologists and are poorly understood. In a world where caffeinated beverages are evidently the adult's drug of choice (coffee, energy drinks, soda, tea) investigating their effects on the physiology of the cardiovascular system is of considerable importance. In this experiment, we investigated caffeine, taken orally as a tablet, on reactive hyperemia, a form of local control of blood flow. Young adults between the ages of 18 and 21 years were the experimental subjects. They were instrumented to monitor systemic arterial blood pressure, peripheral blood flow, calculated peripheral vascular resistance, heart rate and an electrocardiogram during a reactive hyperemia maneuver in the absence and presence of caffeine. Caffeine-mediated peripheral vasoconstriction was observed as early as 15 minutes after its consumption. Forty-five minutes later (60 min after consumption of caffeine) peripheral vasoconstriction was so prominent that reactive hyperemia was abolished. This was reflected, in part, as a marked and significant reduction in post-ischemia reactive hyperemia that accompanied a 2.5 -fold increase in peripheral vascular resistance $(\mathrm{P}<0.05)$. Heart rate was unaffected by caffeine under our experimental conditions. We conclude that caffeine has the ability to inhibit important cardiovascular properties, including reactive hyperemia. If the effects that were seen in a digit are indicative of what caffeine might do in the heart and/or brain, then one has to question the wisdom of regularly consuming caffeine. More experimental physiological and pharmacological investigation is needed.
\end{abstract}

\section{Keywords}

Cardiovascular System, Physiological/Pathophysiological Effects, Caffeine

\section{Introduction}

Caffeine is widely used for medical and non-medical purposes. It seems to have 
some clinical benefits such as respiratory stimulation in premature infants suffering from sleep apnea [1] [2]. However, it is most commonly consumed in beverages and as over-the-counter capsules, pills and tablets because consumers believe it boosts their energy, enhances motivation, improves gym performance, and/or reduces disease [3]-[8]. Thus, abuse of bodies and minds by caffeine has become a pastime for many consumers even though there are unanswered questions about its aphysiological effects on the body, especially the brain and cardiovascular system. For example, since caffeine and other methylxanthines are adenosine receptor inhibitors, adenosine-mediated circulatory regulation in the brain, heart and elsewhere can be dangerously impaired by caffeine [9] [10] [11] [12].

Before the vascular effects of nitric oxide (NO) were discovered [endothelial-derived relaxing factor, EDRF, Furchgott et al. [13] [14], adenosine's ubiquitous role in regulating blood flow to the coronary and other vascular beds [15]-[28] was firmly established (see 15, 19, 28 for Reviews). With the intense interest in NO of the past few decades, most investigators either didn't know this about adenosine or have chosen to ignore it. This does not negate the relevant physiological role adenosine still plays in controlling blood flow under physiological and pathophysiological conditions [15]-[28].

Subsequent to the period of time, circa 1963-1983 (initiation of the investigation of adenosine's physiological roles in the cardiovascular system), exploitation of the public by purveyors of caffeinated products has increased near-exponentially. For example, caffeine is now found in: candy, chewing gum, energy drinks, frozen yogurt, ice cream, sodas, etc. As businesses profit from broad-spread public ignorance it becomes incumbent on physiologists, pharmacologists and similarly-qualified scientists to reveal the pharmacological, pathophysiological and toxicological effects of caffeine and caffeinated products on the human body. It is in the public interest for us to intensify this investigation and to report our results. This study, coupled with our earlier reports [29] [30], represent only one effort towards that end.

\section{Methods}

\subsection{Experimental Subjects}

Experimental subjects were volunteer college-age young adults (male and female) from several cultural/ethnic backgrounds, during Spring Semester, 2019. They were either regular (daily) or irregular (weekly or less frequent) users of caffeinated beverages. All subjects arrived at the laboratory having avoided caffeinated beverages for at least 24 - 48 hours.

\subsection{Instrumentation}

After subjects arrived at the laboratory they were instrumented then rested comfortably on a hospital examination bed in the supine position for $15 \mathrm{mi}$ nutes. Instrumentation included wrapping a sphygmomanometer blood pressure 
cuff, with a built in pressure sensor, around the right ankle. The cuff and pressure sensor were connected to a wall-mounted mercury manometer (Baumanometer, model CE1562). A cardiomicrophone (model MLT201, AD Instruments, Colorado Springs, CO) was placed over a pedal artery on the dorsal surface of the foot and secured in place with Transpore, hypoallergenic surgical tape (3 M Health Care, St. Paul, MN) and Tegaderm Film (3 M Health Care, Neuss, Germany). A pulse plethysmograph (model TN1012/ST, AD Instruments) was attached to the right large or index toe (whichever presented the cleanest signal) and snuggly secured with a Velcro strap. Heart rate was determined from a standard limb lead electrocardiogram (LLI, ECG), and room temperature was maintained at $22^{\circ} \mathrm{C}-24^{\circ} \mathrm{C}$.

\subsection{Data Acquisition System}

All four transducers were attached to a Power Lab data acquisition system (model 8/35, AD Instruments) coupled to a desktop computer (HP Compaq LA2006x) running Lab Chart software (v. 8.1.13). All waveforms could be periodically or continuously displayed on a computer monitor for investigator observation.

Experimental protocol-Following instrumentation a timer was set, lights were dimmed, and a 15-minute period was allowed for monitored variables to achieve physiological steady state conditions. After 15 minutes steady state, baseline data were collected including: recordings of heart rate (HR, cycles per minute, cpm), ECG $(\mathrm{mV})$, systemic mean arterial blood pressure $(\mathrm{Pa}, \mathrm{mmHg})$, and volume in the instrumented toe ( $\mu$ l per pulse). Multiplying pulsatile changes of volume by heart rate yielded an estimate of blood flow $(\mu \mathrm{l} / \mathrm{min})$. Vascular resistance to blood flow in the instrumented digit could then be estimated by dividing mean arterial blood pressure by blood flow $(\mathrm{mmHg} / \mu \mathrm{l} / \mathrm{min})$. Subsequently, the pressure cuff was inflated to $200 \mathrm{mmHg}$ for 30 seconds then rapidly deflated. During inflation blood flow to the instrumented toe was occluded (transient ischemia). Upon deflation blood flow was restored (reactive hyperemia, reperfusion). Data collected during this pre-caffeine period were designated "baseline".

After baseline data were collected each subject consumed $200 \mathrm{mg}$ of caffeine (one tablet NODOZ Alertness Aid, GSK Consumer Healthcare, Warren, NJ). The timer was reset for 60 minutes, and the subjects were asked to rest quietly with as little movement as possible. Hemodynamic variables were monitored by the data acquisition/computer system continuously. However, at 15-minute intervals we estimated blood pressure by inflating/deflating the pressure cuff (20 $30 \mathrm{sec}$ ). Restoring blood flow to the toe (post-occlusion of the compressed pedal artery) and monitoring pulsatile arterial sounds (cardiomicrophone) could be used to estimate pulsatile and mean arterial blood pressures at these 15-minute timed intervals. At 60 minutes post-caffeine the ischemia/reperfusion (reactive hyperemia) maneuver was again completed. Data collected during this period were considered "experimental" and were later compared, statistically, with the pre-caffeine data. 


\subsection{Statistics}

The experiment was designed a priori and is a follow up to related experiments conducted in our laboratory [13] [14]. Initial variability was identified using Analysis of Variance for Repeated Measures (one-way ANOVA). Means were compared using Students t-test. All data are presented as means plus or minus one standard error of the mean (s.e.m.). Statistically significant differences were established at $\mathrm{P}<0.05$.

\section{Results}

Figure 1 illustrates an example of an entire experiment compressed horizontally. Routinely, 60 minutes after an oral dose of $200 \mathrm{mg}$ caffeine, all subjects experienced a marked and statistically significant reduction in digit blood flow. This was accompanied by the inability of that digit to regulate its blood supply during a brief period of ischemia and reperfusion (i.e. reactive hyperemia was abolished). By examining Figure 1, the reader can see that in the absence of caffeine, blood flow increased about $50 \%-60 \%$ above its baseline value following release of a 30 -sec period of occlusion of flow through a pedal artery. In the presence of caffeine, post-ischemia, reactive hyperemia did not occur.

\begin{tabular}{|ll|}
\hline before caffeine & after caffeine \\
\hline
\end{tabular}

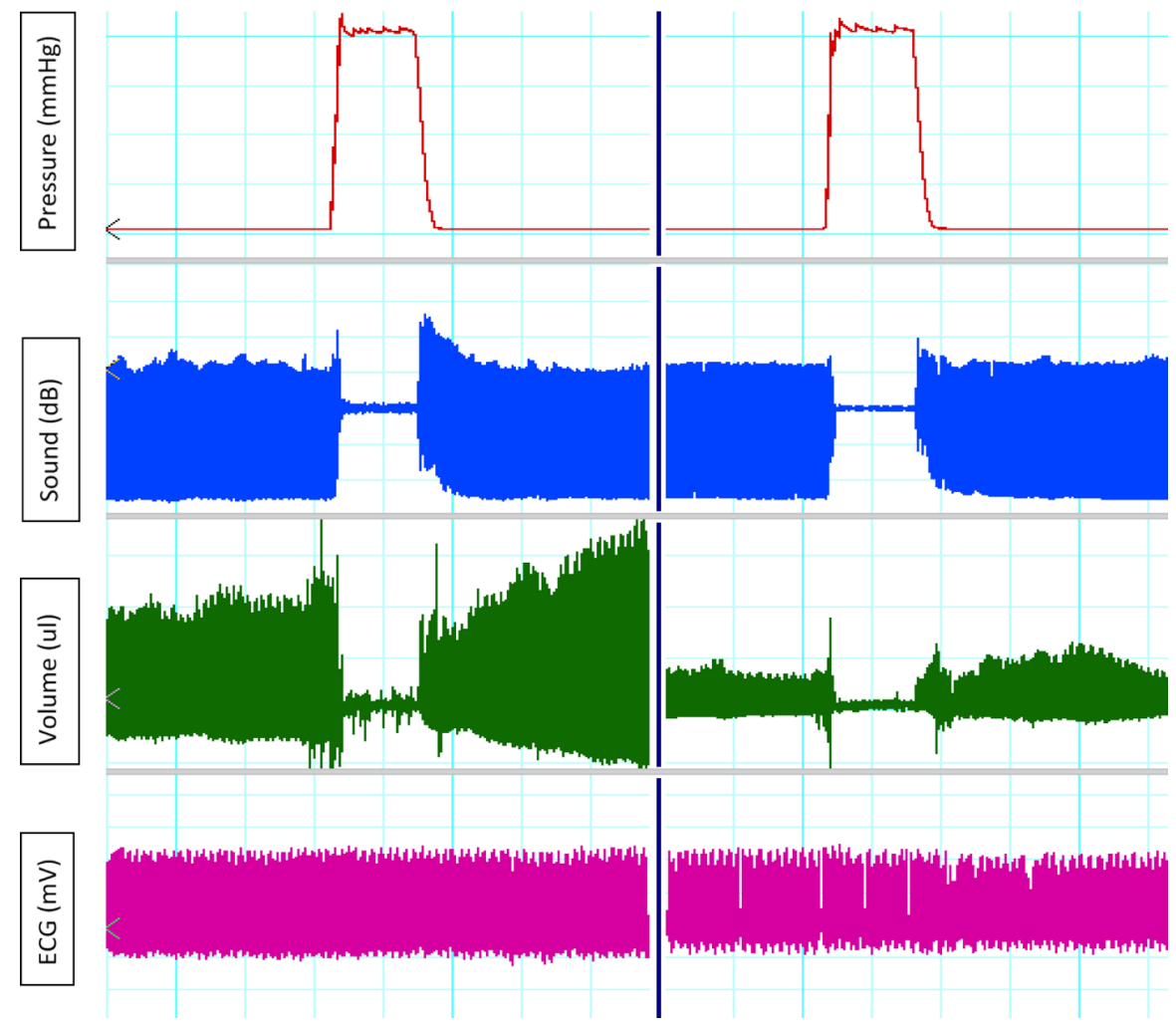

Figure 1. Sample tracing of reactive hyperemia before and 60 minutes after oral consumption of $200 \mathrm{mg}$ caffeine. Note the significant decrease in ability of the peripheral vasculature to regulate its blood supply in the presence of caffeine. 
In the absence of caffeine, blood flow to the toe increased from a baseline value of $2579 \pm 487 \mu \mathrm{l} / \mathrm{min}$ to a peak hyperemic flow of $3892 \pm 647 \mu \mathrm{l} / \mathrm{min}(\mathrm{P}<$ $0.05)$. Corresponding values in the presence of caffeine were $1279 \pm 332 \mu \mathrm{l} / \mathrm{min}$ and $1343 \pm 295 \mu \mathrm{l} / \mathrm{min}$ (statistically insignificant, see Figure 2). Reactive hyperemia can be quantified in many ways. Another expression of reactive hyperemia is the duration of the flow response following release of an occlusion; also the time it takes for the maximum flow to be achieved (studied here). In our experiment, and in the absence of caffeine, the time required for flow to reach a maximum value was $114 \pm 15$ seconds. Corresponding numbers 60 minutes after $200 \mathrm{mg}$ of caffeine were $50 \pm 11$ seconds $(\mathrm{P}<0.05)$ (Figure 3 ).

Resistance to blood flow was $0.05 \pm 0.01 \mathrm{mmHg} / \mu \mathrm{l} / \mathrm{min}$ before caffeine administration. Sixty minutes after caffeine, resistance to blood flow was elevated markedly and significantly to $0.12 \pm 0.03 \mathrm{mmHg} / \mu \mathrm{l} / \mathrm{min}(\mathrm{P}<0.05)$. This nearly 2.5 -fold increase in caffeine-mediated vasoconstriction explains the inability of the vasculature to regulate its blood supply during and after a brief period of ischemia (Figure 4).

Systemic arterial blood pressure, whether expressed as systolic, diastolic or mean pressure was significantly elevated by caffeine (Figure 5). The increase in pressure, accompanied by an increase in resistance to flow and corresponding decrease in flow, were seen as early as 15 - 30 minutes in many subjects; it was evident in all subjects after 30 minutes and reached its zenith at $60 \mathrm{mi}$ nutes.

Heart rate did not change significantly in the presence of $200 \mathrm{mg}$ caffeine. This was true regardless of the time interval between $1-60$ minutes post-consumption of caffeine. We did not scrutinize the ECG for significant ectopy. However, we have

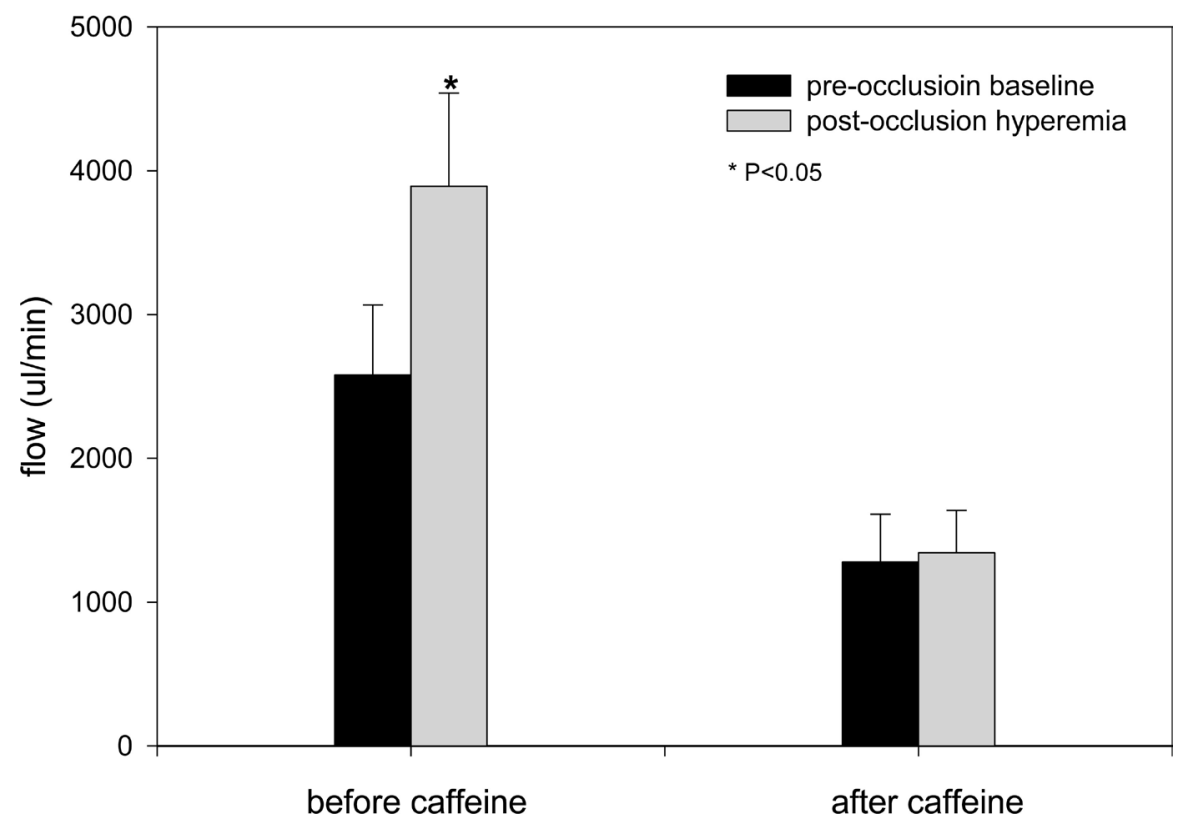

Figure 2. Change in blood to the toe during reactive hyperemia before and 60 minutes after oral consumption of $200 \mathrm{mg}$ caffeine. 


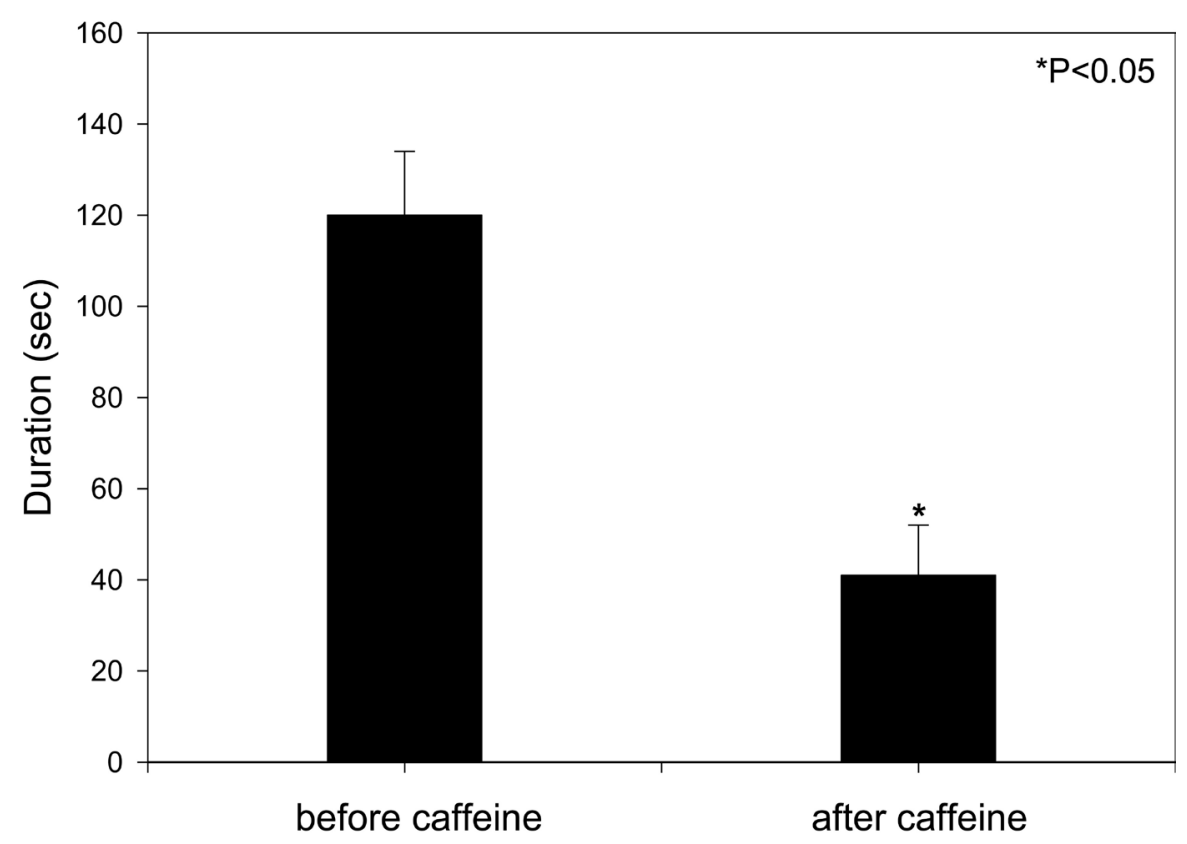

Figure 3. Duration of reactive hyperemia before and 60 minutes after oral consumption of $200 \mathrm{mg}$ caffeine.

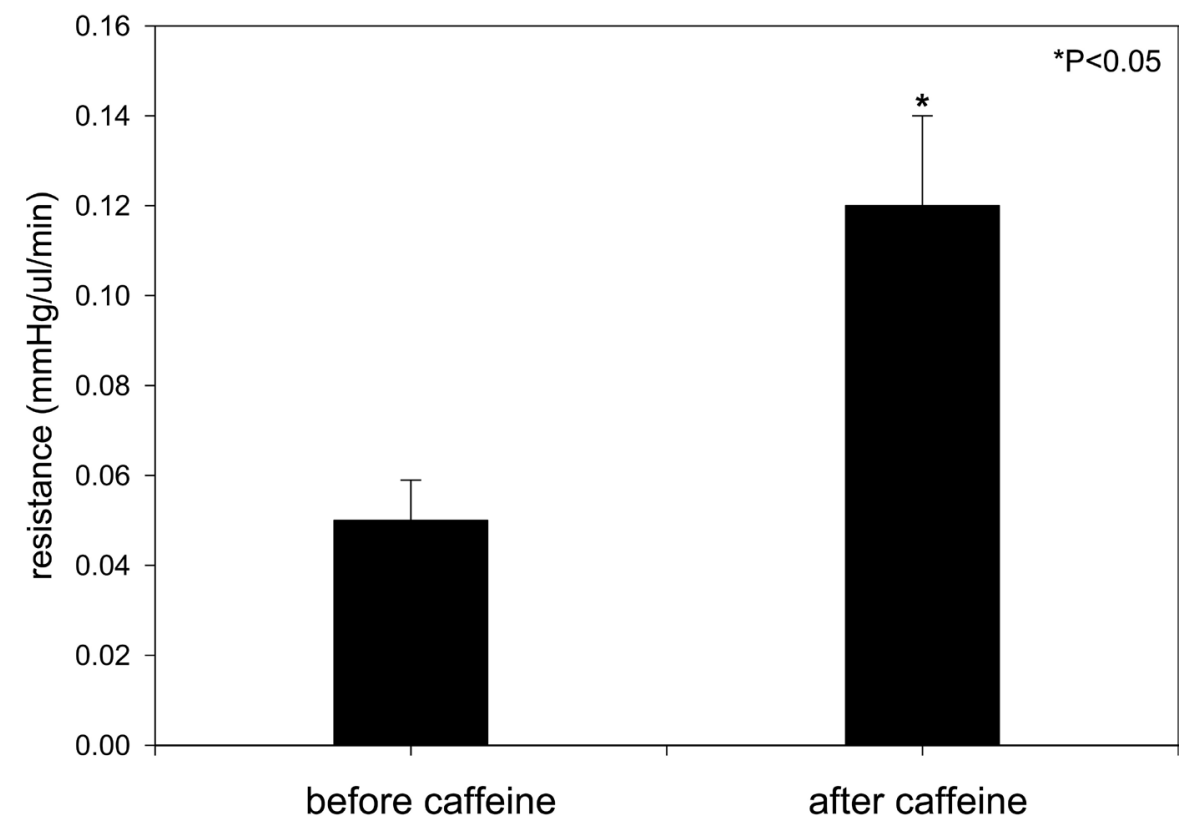

Figure 4. Significantly increased peripheral vascular resistance (toe) 60 minutes after oral consumption of $200 \mathrm{mg}$ caffeine.

frequently seen wandering baselines (i.e. an unstable isoelectric line) and high amplitude $\mathrm{T}$ waves within 15 - 30 minutes of caffeine consumption. This is true whether the drug is taken as an oral tablet/capsule or in favorite hot drinks (e.g. coffee). These aphysiological, electrical effects have been occasionally accompanied by ventricular premature beats (VPB), ventricular salvos (VS) and deviated ST-segments. 


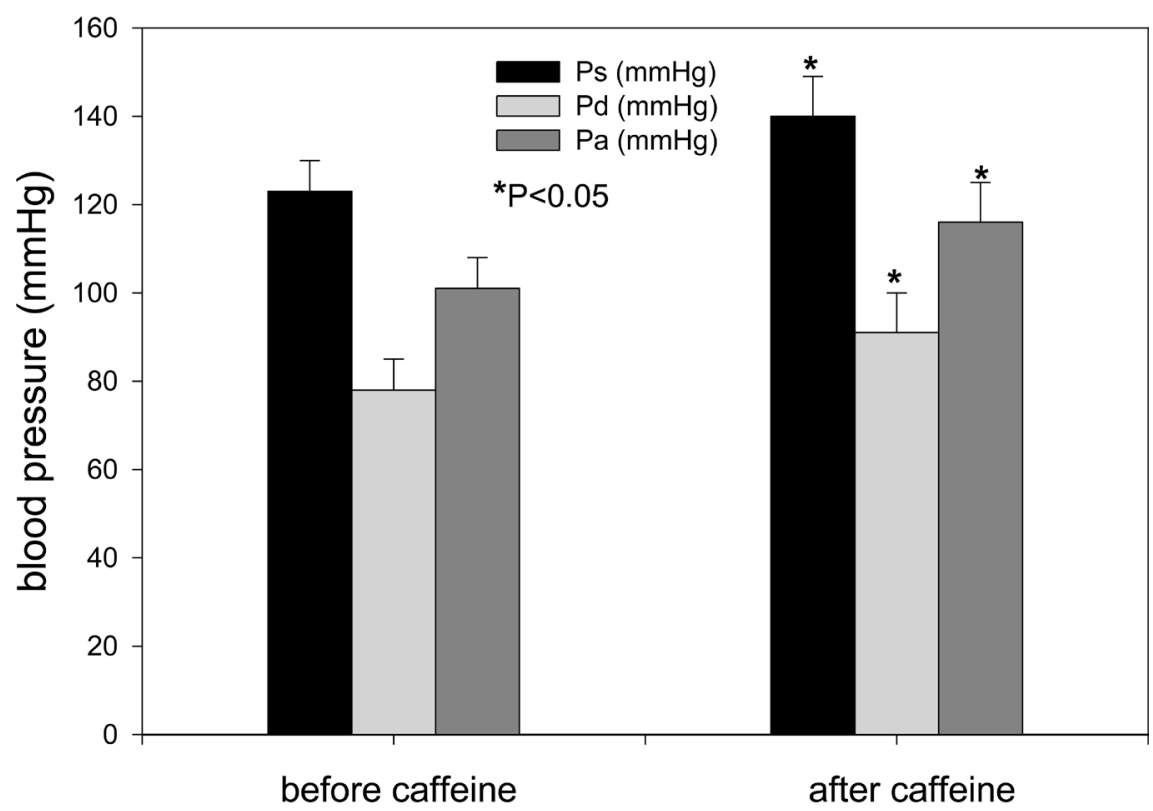

Figure 5. Significant increase in systolic, diastolic and mean systemic arterial blood pressure 60 minutes following oral consumption of $200 \mathrm{mg}$ caffeine.

\section{Discussion}

According to the Nutrient Data Laboratory, National Nutrient Database (USDA, Human Nutrition Research Center, 10,300 Baltimore Ave., Beltsville, MD) one 8-ounce cup of coffee ( 240 milliliters) contains about 100 milligrams of caffeine. Of some fifty three sports drinks and dietary supplements products analyzed by the USDA in 2004-05, twenty seven products provided the caffeine equivalent of about one to two cups of coffee ( $100-200 \mathrm{mg}$, per manufacturers labels), eleven products had caffeine equivalents ranging from two to four cups of coffee ( 200 - $400 \mathrm{mg}$ ), another eleven had equivalents ranging from four to six cups of coffee ( 400 - $600 \mathrm{mg})$, and four products provided an amount of caffeine ranging from seven to eight cups of coffee $(\sim 700-800 \mathrm{mg})$.

In our previous experiments we have found that $200-400 \mathrm{mg}$ of caffeine disturbs the isoelectric line in standard limb lead ECGs and causes statistically significant elevations in peripheral vascular resistance that are accompanied by marked and significant reductions in blood flow and increments in systemic arterial blood pressure. These same doses of caffeine also significantly block peripheral vascular pressure-flow autoregulation [13] [14]. As yet we have not examined the detailed effects of caffeine on the intervals, segments and waves of standard limb lead ECGs.

\subsection{Caffeine and Reactive Hyperemia}

For many years scientists and others have had an interest in the regulation of blood flow to the extremeties [31] [32] [33]. This interest is driven, in part, by conditions such as Raynaud's syndrome and from construction/lumbering industries where the mechanical agitation of hands and digits are an integral com- 
ponent of the work. In both clinical and environmental settings blood flow to the digits can be affected by changes in temperature and vibration. These and related findings have also prompted investigators to examine the influence of work and environment on reactive hyperemia of the digits [34] [35] [36]. However, reactive hyperemia is only one important component of the local regulation of blood flow. Other components include, but are not limited to, pressure-flow autoregulation and exercise hyperemia (or active hyperemia).

Within 15 - 30 minutes after administration of $200 \mathrm{mg}$ caffeine (orally) statistically significant peripheral vasoconstriction was observed in the toes of all experimental subjects in the current investigation. The vasoconstriction and accompanying elevations in peripheral vascular resistance and arterial perfusion pressure, as well as reduced blood flow, intensified in the next 30 - 45 minutes. These results are consistent with earlier experimental investigations from which we have reported significant caffeine-mediated peripheral vasoconstrictor effects in humans. In two of the three studies ([14], current report) we have observed caffeine-mediated blockade of local blood flow-regulating mechanisms, i.e. pressure-flow autoregulation [14] and reactive hyperemia (current report).

Even though we did not investigate physiological mechanisms in this experiment, it is both logical and reasonable to implicate the adenosine hypothesis and caffeine's interference with the vascular effects of adenosine as the cause of these caffeine-mediated effects. Briefly, the adenosine hypothesis reveals that the local release of adenosine during periods of tissue ischemia and hypoxia results in the subsequent maintenance or improvement of blood flow to the affected tissues/organs [16] [17] [18] [24]-[30]. If the blood supply to an organ or tissue is significantly reduced by vasoconstriction, then the organ/tissue is ischemic. If adenosine cannot bind to its vascular receptors (due to caffeine blockade) then it cannot restore blood flow.

\subsection{Adenosine and Blood Flow}

Before the vascular effects of nitric oxide (NO) were discovered [endothelial-derived relaxing factor, EDRF, Furchgott et al. [37] [15], adenosine's ubiquitous role in regulating blood flow to the coronary and other vascular beds [16]-[29] was firmly established (see [16] [20] [29]). With the intense interest in NO of the past few decades, most investigators either do not know this about adenosine or have chosen to ignore it. This does not negate the relevant physiological role adenosine still plays in controlling blood flow under physiological and pathophysiological conditions [16]-[29].

Subsequent to the period, circa1963-1983 (initiation of the investigation and establishment of adenosine's physiological roles in the cardiovascular system), exploitation of public ignorance by the caffeinated-products industry has increased near-exponentially. For example, today (2019) caffeine can be found in: candy, chewing gum, energy drinks, frozen yogurt, ice cream, sodas, and who-knows-what else. Before circa 1963 its primary consumption was in the form of coffee, colas, chocolate and tea. As the caffeinated-products industry and oth- 
er businesses profit from public confusion and ignorance it becomes incumbent on physiologists, pharmacologists and similarly-qualified life scientists to help reveal the pharmacological, pathophysiological and toxicological effects of caffeine and caffeinated products on the human body.

Mechanistically, one line of future investigation could be the pharmacological construction of peripheral vascular adenosine dose-response curves in the absence and presence of caffeine. Similar studies can and should be done using caffeine's closest congeners theobromine, theophylline and related compounds (e.g. aminophylline).

\section{Limitations, Summary and Conclusions}

In the current study, we did not monitor cardiovascular variables beyond sixty minutes. Still, we know that caffeine and its byproducts are metabolized, washed out and excreted in several hours depending on the individual and dose of caffeine [3] [4]. Notwithstanding, sixty minutes is adequate time for caffeine to demonstrate its previously-unreported, aphysiological_even pathophysiological_effects on the human cardiovascular system. These effects have been seen after consumption of hot coffee [13] [38], and with oral doses of the drug (tablet/capsule forms) ranging from 200 - $400 \mathrm{mg}$ [14].

The drug of choice for the world's adult population is caffeine. Locally, this can be confirmed almost any week day on university campuses where food trucks park near academic buildings and long lines of students wait to get their morning/afternoon Joe. Similar observations can be made at building construction sites mid-morning, near-noon and early-afternoon as workers stop to get their daily fixes of caffeine. Nonexperimental, even pseudoscientific analyses have extolled the potential benefits of caffeine. Nonetheless, there has been a paucity of experimental investigations by physiologists, pharmacologists and toxicologists on the pathophysiological effects of the drug. In our experimental, cardiovascular physiology laboratory, we have recently reported that caffeine blocks digital pressure-flow autoregulation. Here we report caffeine's ability to attenuate digital reactive hyperemia. If caffeine blocks the regulation of blood flow in fingers and toes, one must ask, what is caffeine doing in the cerebral, coronary, renal, and other more important vascular beds.

\section{Conflicts of Interest}

The authors declare no conflicts of interest regarding the publication of this paper.

\section{References}

[1] Funk, G.D. (2009) Losing Sleep over the Caffeination of Prematurity. Journal of Physiology, 587, 5299-5300. https://doi.org/10.1113/jphysiol.2009.182303

[2] Doyle, L.W., Ranganathan, S. and Cheong, J.L.Y. (2017) Neonatal Caffeine Treatment and Respiratory Function at Eleven Years in Children under 1,251 g at Birth. American Journal of Respiratory and Critical Care Medicine, 196, 1318-1324. 
https://doi.org/10.1164/rccm.201704-0767OC

[3] Doepker, C., Franke, K., Myers, E., Goldberger, J.J., Lieberman, H.R., O’Brien, C., Peck, J., Tenenbein, M., Weaver, C. and Wikoff, D. (2018) Key Findings and Implications of a Recent Systematic Review of the Potential Adverse Effects of Caffeine Consumption in Healthy Adults, Pregnant Women, Adolescents, and Children. Nutrients, 10, 1536-1552. https://doi.org/10.3390/nu10101536

[4] Voskoboinik, A., Koh, Y. and Kistler, P.M. (2019) Cardiovascular Effects of Caffeinated Beverages. Trends in Cardiovascular Medicine, 29, 345-350. https://doi.org/10.1016/j.tcm.2018.09.019

[5] Cappelletti, S., Piacentino, D., Sani, G. and Aromatario, M. (2015) Caffeine: Cognitive and Physical Performance Enhancer of Psychoactive Drug? Current Neuropharmacology, 13, 71-88. https://doi.org/10.2174/1570159X13666141210215655

[6] Ferre, S. (2016) Mechanisms of the Psychostimulant Effects of Caffeine: Implications for Substance Use Disorders. Psychopharmacology, 233, 1963-1979. https://doi.org/10.1007/s00213-016-4212-2

[7] Grasser, E.K., Miles-Chan, J.L., Charriere, N., Loonam, C.R., Dulloo, A.G. and Montani, J.P. (2016) Energy Drinks and Their Impact on the Cardiovascular System: Potential Mechanisms. Advances in Nutrition, 7, 950-960.

https://doi.org/10.3945/an.116.012526

[8] McLellan, T.M., Caldwell, J.A. and Lieberman, H.R. (2016) A Review of Caffeine's Effects on Cognitive, Physical and Occupational Performance. Neuroscience \& Biobehavioral Reviews, 71, 294-312. https://doi.org/10.1016/j.neubiorev.2016.09.001

[9] Urry, E. and Landolt, H.P. (2015) Adenosine, Caffeine, and Performance: From Cognitive Neuroscience of Sleep to Sleep Pharmacogenetics. Current Topics in Behavioral Neurosciences, 25, 331-366.

[10] Rivera-Oliver, M. and Diaz-Rios, M. (2014) Using Caffeine and Other Adenosine Receptor Antagonists and Agonists as Therapeutic Tools against Neurodegenerative Diseases. Life Sciences, 101, 1-9. https://doi.org/10.1016/j.lfs.2014.01.083

[11] Boia, R., Ambrosio, A.F. and Santiaago, A.R. (2016) Therapeutic Opportunities for Caffeine and A2a Receptor Antagonists in Retinal Diseases. Ophthalmic Research, 55, 212-218. https://doi.org/10.1159/000443893

[12] Ferre, S., Bonaventura, J., Tomasi, D., Navarro, G., Moreno, E., Cortex, A., Liuis, C., Casado, V. and Volkow, N.D. (2016) Allosteric Mechanisms within the Adenosine A2a Dopamine D2 Receptor Heterotetramer. Neuropharmacology, 104, 154-160. https://doi.org/10.1016/j.neuropharm.2015.05.028

[13] Merrill, G.F. and Sharp, V.A. (2018) Undesirable Cardiovascular Effects of Hot Drinks. International Journal of Clinical and Medical Cases, 1, 117-124.

[14] Merrill, G.F., Costea, D.M. and Sharp, V.A. (2019) Caffeine and Pressure Flow Autoregulation. World Journal of Cardiovascular Diseases, 9, 253-266. https://doi.org/10.4236/wjcd.2019.94023

[15] Furchgott, R.F., Zawadzki, J.V., Jothianandan, D. and Cherry, P.D. (1982) Role of Endothelial Cells in Relaxation of Isolated Arteries by Bradykinin. Proceedings of the National Academy of Sciences of the United States of America, 79, 2106-2110. https://doi.org/10.1073/pnas.79.6.2106

[16] Berne, R.M., Winn, H.R. and Rubio, R. (1981) The Local Regulation of Cerebral Blood Flow. Progress in Cardiovascular Diseases, 24, 243-260. https://doi.org/10.1016/0033-0620(81)90030-X

[17] Olsson, R.A. (1981) Local Factors Regulating Cardiac and Skeletal Muscle Blood 
Flow. Annual Review of Physiology, 43, 385-395. https://doi.org/10.1146/annurev.ph.43.030181.002125

[18] Berne, R.M. (1980) The Role of Adenosine in the Regulation of Coronary Blood Flow. Circulation Research, 47, 807-813. https://doi.org/10.1161/01.RES.47.6.807

[19] Mustafa, S.J. (1980) Cellular and Molecular Mechanism(s) of Coronary Flow Regulation by Adenosine. Molecular and Cellular Biochemistry, 31, 67-87. https://doi.org/10.1007/BF00240813

[20] Feigl, E.O. (1983) Coronary Physiology. Physiological Reviews, 63, 1-205.

[21] Haddy, F.J. (1977) Bioassay and Pharmacologic Evaluation of the Adenosine Hypothesis. Advances in Experimental Medicine and Biology, 78, 175-182.

[22] Burnstock, G. (1977) The Purinergic Nerve Hypothesis. Ciba Foundation Symposium, 48, 295-314.

[23] Verhaeghe, R.H., Lorenze, R.R., McGrath, M.A., Shepherd, J.T. and Vanhoutte, P.M. (1978) Metabolic Modulation of Neurotransmitter Release-Adenosine, Adenine Nucleotides, Potassium, Hyperosmolarity, and Hydrogen Ion. Federation Proceedings, 37, 208-211.

[24] Rubio, R. and Berne, R.M. (1975) Regulation of Coronary Blood Flow. Progress in Cardiovascular Diseases, 18, 105-122. https://doi.org/10.1016/0033-0620(75)90001-8

[25] Berne, R.M. and Rubio, R. (1974) Regulation of Coronary Blood Flow. Advances in Cardiology, 12, 303-317.

[26] Haddy, F.J. (1969) Physiology and Pharmacology of the Coronary Circulation and Myocardium, Particularly in Relation to Coronary Artery Disease. The American Journal of Medicine, 47, 274-286. https://doi.org/10.1016/0002-9343(69)90153-3

[27] Rubio, R. and Berne, R.M. (1969) Release of Adenosine by the Normal Myocardium in Dogs and Its Relationship to the Regulation of Coronary Resistance. Circulation Research, 25, 407-415. https://doi.org/10.1161/01.RES.25.4.407

[28] Rubio, R., Berne, R.M. and Katori, M. (1969) Release of Adenosine in Reactive Hyperemia of the Dog Heart. American Journal of Physiology, 216, 56-62.

[29] Berne, R.M. (1964) Regulation of Coronary Blood Flow. Physiological Reviews, 44, 1-29.

[30] Berne, R.M. (1963) Cardiac Nucleotides in Hypoxia: Possible Role in Regulation of Coronary Blood Flow. American Journal of Physiology, 204, 317-329.

[31] Ye, Y., Mauro, M., Bovenzi, M. and Griffin, M.J. (2015) Reduction in Finger Blood Flow Induced by Hand-Transmitted Vibration: Effect of Hand Elevation. International Archives of Occupational and Environmental Health, 88, 981-992. https://doi.org/10.1007/s00420-015-1027-0

[32] Trager, S., Pignataro, M., Anderson, J. and Kleinert, J.M. (1993) Color Flow Doppler: Imaging the Upper Extremity. Journal of Hand Surgery, 18, 621-625. https://doi.org/10.1016/0363-5023(93)90304-L

[33] Goodfield, M., Hume, A. and Rowell, N. (1989) Reactive Hyperemic Responses in Systemic Sclerosis Patients and Healthy Controls. Journal of Investigative Dermatology, 93, 368-371.

[34] McDonnell, A.C., Eiken, O., Mekjavic, P.J. and Mekjavic, I.B. (2014) Circadian Rhythm of Peripheral Perfusion during 10-Day Hypoxic Confinement and Bed Rest. European Journal of Applied Physiology, 114, 2093-2104.

https://doi.org/10.1007/s00421-014-2923-9 
[35] Yan, Y., Shen, G., Xie, K., Tang, C., Wu, X., Xu, Q., Liu, J., Song, J., Jiang, X. and Luo, E. (2011) Wavelet Analysis of Acute Effects of Static Magnetic Field on Resting Skin Blood Flow at the Nail Wall in Young Men. Microvascular Research, 82, 277-283. https://doi.org/10.1016/j.mvr.2011.03.008

[36] Macefield, V.G. (2003) Cardiovascular and Respiratory Modulation of Tactile Afferents in the Human Finger Pad. Experimental Physiology, 88, 617-625. https://doi.org/10.1113/eph8802548

[37] Furchgott, R.F. and Zawadzki, J.V. (1980) The Obligatory Role of Endothelial Cells in the Relaxation of Arterial Smooth Muscle by Acetylcholine. Nature, 288, 373-376. https://doi.org/10.1038/288373a0

[38] Merideth, A., Addicott, A., Yang, L.L., Peiffer, A.M., et al. (2009) The Effect of Daily Caffeine Use on Cerebral Blood Flow: How Much Caffeine Can We Tolerate? Human Brain Mapping, 30, 3102-3114. https://doi.org/10.1002/hbm.20732 